\title{
BMJ Open Antithrombotic regimens in females with symptomatic lower extremity peripheral arterial disease: protocol for a systematic review and meta-analysis
}

Shira A Strauss (D) , ${ }^{1,2}$ Prasad Jetty, ${ }^{1,2,3}$ Daniel Kobewka, ${ }^{2,3,4,5}$ Marc Carrier ${ }^{2,3,4,5}$

To cite: Strauss SA, Jetty P, Kobewka D, et al. Antithrombotic regimens in females with symptomatic lower extremity peripheral arterial disease: protocol for a systematic review and meta-analysis. BMJ Open 2021;11:e042980. doi:10.1136/ bmjopen-2020-042980

- Prepublication history and additional supplemental material for this paper are available online. To view these files, please visit the journal online (http://dx.doi.org/10.1136/ bmjopen-2020-042980)

Received 22 July 2020 Revised 23 March 2021 Accepted 29 April 2021
Check for updates

(C) Author(s) (or their employer(s)) 2021. Re-use permitted under CC BY-NC. No commercial re-use. See rights and permissions. Published by BMJ.

For numbered affiliations see end of article.

Correspondence to

Dr Shira A Strauss;

shira.strauss@medportal.ca

\section{ABSTRACT}

Introduction Patients with peripheral arterial disease

(PAD) are at increased risk for systemic arterial thromboembolic events. Females represent a unique subset of patients with PAD, who differ from males in important ways: they have smaller diameter vessels, undergo lower extremity bypass less frequently and experience higher rates of graft occlusion, amputation and mortality than males. Females also trend towards higher rates of major coronary events and cardiovascular mortality. Current guidelines recommend monoantiplatelet therapy (MAPT) for secondary prevention in patients with symptomatic PAD. However, indications for more intensive antithrombotic therapy in this cohort-especially among females who are frequently under-represented in randomised controlled trials (RCTs) - remain unclear. As newer antithrombotic therapies emerge, some RCTs have demonstrated differential effects in females versus males. A systematic review is needed to quantify the rates of arterial thromboembolic and bleeding events with different antithrombotic regimens in females with symptomatic PAD.

Methods and analysis We will search MEDLINE, Embase and the Cochrane Central Register of Controlled trials for published RCTs that include females with symptomatic PAD and compare full dose anticoagulation \pm antiplatelet therapy, dual pathway inhibition or dual antiplatelet therapy with MAPT. Title, abstract and full-text screening will be conducted in duplicate by three reviewers. Authors will be contacted to obtain sex-stratified outcomes as needed. Risk of bias will be assessed using the Cochrane Risk of Bias tool. Data will be extracted by independent reviewers and confirmed by a second reviewer. Quantitative synthesis will be conducted using Review Manager (RevMan) V.5 for applicable outcomes data. Planned subgroup analysis by PAD severity, vascular intervention and indication for antithrombotics will be conducted where data permits.

Ethics and dissemination Ethics approval is waived as the study does not involve primary data collection. This review will be submitted for publication in a peer-reviewed journal and for presentation at national and international scientific meetings.

Trial registration number This protocol was registered with the PROSPERO International ProspectiveRegister of Systematic Reviews (ID\# CRD42020196933).

\section{Strengths and limitations of this study}

- This protocol was reported in accordance with the Preferred Reporting Items for Systematic Review and Meta-Analysis Protocols guidelines.

- This review offers a broad search strategy to capture all studies that might include the population of interest, including as a subset of a larger sample.

- This review addresses an important gap in the literature by targeting female-specific vascular outcomes.

- This review will not be limited by language or date specifications.

Depending on the availability of sex-stratified data, quantitative synthesis may not be possible for all outcomes of interest.

\section{INTRODUCTION}

\section{Rationale}

Lower extremity peripheral arterial disease (PAD) is common, affecting approximately 800000 Canadians and over 200 million people worldwide. ${ }^{12}$ Patients with large-vessel PAD are at increased risk for arterial thromboembolic events compared with those without, with a 5.9-fold increased risk of death from all cardiovascular (CV) causes and a 6.6-fold increased risk of death from coronary heart disease. ${ }^{3}$ Among patients with severe, untreated PAD, 1-year mortality and amputation rates reach $22 \% .{ }^{4}$ While previous demographic data have yielded mixed findings about the distribution of PAD across sexes, ${ }^{5-9}$ more recent studies have revealed the prevalence to be at least equal, if not higher, in females compared with males. ${ }^{10}$ Despite similar distributions of the disease, females with PAD differ from males in a number of important ways, ${ }^{10-12}$ including higher functional impairment, lower likelihood of undergoing lower extremity bypass and, potentially, higher bleeding risk. ${ }^{13-19}$ Among females who do undergo lower extremity bypass, rates of graft occlusion, 
amputation and mortality are higher than in males. ${ }^{16} 20$ Females with PAD also trend towards higher rates of $\mathrm{CV}$ mortality and major coronary events as compared with their male counterparts. ${ }^{10}$ Smaller diameter vessels, older age at presentation, hormonal differences and greater number of comorbidities may explain the discrepancy in treatment, risks and outcomes between the sexes. ${ }^{11} 19$

To mitigate the risk of arterial thromboembolic events, antithrombotic therapy has become a fundamental component of the management of symptomatic PAD. ${ }^{21} 22$ Antithrombotic therapy refers to antiplatelet agents and/ or anticoagulants, which target different steps in the coagulation cascade. Antiplatelet agents interfere with platelet activation, adhesion and aggregation through various mechanisms, such as thromboxane A1 inhibition (aspirin), ADP receptor antagonism (P2Y ${ }_{12}$ inhibitors such as clopidogrel) and glycoprotein IIb/IIIa inhibition (eg, abciximab). ${ }^{23}$ In contrast, anticoagulants primarily affect secondary haemostasis by targeting components of the coagulation cascade. For example, vitamin $\mathrm{K}$ antagonists, such as warfarin, impair the activity of vitamin $\mathrm{K}$ dependent clotting factors, ${ }^{23}$ and direct oral anticoagulants, such as dabigatran and rivaroxaban, inhibit factor IIa (thrombin) and factor Xa, respectively. ${ }^{24} 25$ Finally, dual pathway inhibition (DPI), which combines very low dose anticoagulation (typically rivaroxaban) with antiplatelet therapy (typically aspirin), is thought to synergistically reduce thromboembolic risk by inhibiting platelet activation and attenuating thrombin generation. ${ }^{26}$ Careful consideration of the patient's comorbidities, characteristics, intrinsic thrombogenicity and indication for-as well as response to-antithrombotic therapy must guide selection of the appropriate regimen.

The benefits of antiplatelet therapy are well documented in the Antithrombotic Trialists' Collaboration meta-analysis, which demonstrated a significant reduction in non-fatal stroke, non-fatal myocardial infarction (MI) and CV death with antiplatelet therapy in patients at high risk for vascular occlusive events $(p=0.004) .^{27}$ However, with more intensive antithrombotic therapy, there is ambiguity surrounding which PAD populations benefit most. The CHARISMA trial, which compared dual antiplatelet therapy (DAPT) with aspirin alone, revealed no reduced risk in the $\mathrm{PAD}$ subgroup with respect to the composite outcome: CV death, MI or stroke. ${ }^{28}$ Likewise, in the CASPAR trial, DAPT did not significantly reduce the composite outcome of index graft occlusion or revascularisation, ipsilateral above-ankle amputation or death compared with aspirin alone in patients with recent belowknee bypass for PAD. ${ }^{29}$ However, in another CHARISMA subgroup analysis that combined patients with prior MI, stroke or symptomatic PAD, DAPT significantly reduced rates of the composite outcome in patients with atherosclerotic disease in multiple vascular beds. ${ }^{30}$ In PAD patients undergoing lower extremity endovascular revascularisation, a number of trials have compared DAPT with monoantiplatelet therapy (MAPT), with no clear consensus about its benefits. ${ }^{31-33}$
There are also several trials that examined the use of full dose anticoagulation in patients with PAD. One demonstrated that anticoagulation in addition to antiplatelet therapy did not reduce rates of $\mathrm{MI}$, stroke or $\mathrm{CV}$ death in patients with symptomatic $\mathrm{PAD}^{34}$; one demonstrated that anticoagulation did not reduce rates of graft occlusion compared with aspirin in patients who underwent infrainguinal bypass grafting ${ }^{35}$; one demonstrated that anticoagulation increased graft patency at the cost of increased perioperative haematoma in high-risk patients undergoing vein bypass grafting ${ }^{36}$; and a few additional studies examining different anticoagulation versus antiplatelet comparisons in PAD patients offered no conclusive evidence. ${ }^{37}$ The question of whether particularly high-risk PAD patients-such as those undergoing bypass therapy with a specific type of graft—may benefit from the addition of full dose anticoagulation remains.

Recently, DPI has emerged as an antithrombotic option in patients at high risk for CV events. In particular, very low dose $(2.5 \mathrm{mg}$ two times per day) rivaroxaban in combination with aspirin gained popularity after publication of the COMPASS trial, which demonstrated a reduction in major adverse cardiac events (CV death, stroke and MI) with DPI compared with aspirin alone in patients with stable CV disease. ${ }^{38}$ This study led to its approval in Canada for secondary prevention in patients with coronary artery disease or symptomatic, high-risk PAD. ${ }^{39}$ The VOYAGER trial followed, demonstrating a reduction in the composite outcome of acute limb ischaemia, major amputation for vascular causes, MI, ischaemic stroke or CV death with the addition of very low dose rivaroxaban to aspirin in PAD patients with recent lower extremity revascularisation. ${ }^{40}$ While no heterogeneity was observed in the efficacy of DPI between sexes, sex-stratified analyses revealed that neither the primary efficacy nor safety outcome was statistically significant in females. Since the trial was not powered to detect a difference in the female subgroup, it can be difficult to interpret the clinical significance of these findings. Pooling the data from similar trials may uncover interesting trends according to sex and inform clinical practice guidelines, which are currently in evolution. $^{41}$

The existing data have led to uncertainty about optimal antithrombotic therapy in patients with PAD; however, the ambiguity is even more pronounced within the female subpopulation. Despite differences between males and females with PAD, ${ }^{10-17}$ females are consistently underrepresented in vascular trials. ${ }^{42} 43$ Females have also demonstrated different responses than males to vascular interventions in other vascular beds; for example, males with asymptomatic carotid stenosis derived benefit from carotid endarterectomy while females did not. ${ }^{44}$ Yet, sex is not currently factored into decision-making algorithms for antithrombotic therapy for PAD in clinical practice guidelines. $^{22} 4145$ In 2012, the American Heart Association released a statement that highlighted the differences between males and females with PAD and stressed the need for sex-specific CV research. ${ }^{10}$ This study aims to 
respond to that call by systematically reviewing the literature on rates of arterial thromboembolic and bleeding events with different antithrombotic regimens in females with symptomatic PAD.

\section{Objective}

To systematically review the literature reporting efficacy and safety outcomes of various antithrombotic regimens with respect to arterial thromboembolic and bleeding events in females with symptomatic PAD.

\section{METHODS}

\section{Protocol and registration}

This protocol was reported in accordance with the Preferred Reporting Items for Systematic Reviews and Meta-Analysis Protocols (PRISMA-P) guidelines (see online supplemental appendix A for PRISMA-P Reporting Checklist). ${ }^{46}$ The protocol was registered with the PROSPERO International Prospective Register of
Systematic Reviews (ID\# CRD42020196933). If changes to the protocol are required, we will report deviations from the original protocol and rationale with transparency.

\section{Eligibility criteria}

Inclusion and exclusion criteria are presented in table 1.

\section{Information sources}

We will search MEDLINE, Embase and the Cochrane Central Register of Controlled trials. The information sources will be supplemented by searching registered clinical trials on ClinicalTrials.gov and the International Standard Randomised Controlled Trials Number database. Grey literature will be excluded. We will also scan the reference list of systematic reviews of similar scope and included trials to ensure that all relevant studies are captured. If sex-stratified outcomes are not reported for studies that otherwise meet inclusion criteria, authors will be contacted to request sex-stratified data.

\begin{tabular}{|c|c|c|}
\hline & Inclusion & Exclusion \\
\hline Study design & $\begin{array}{l}\text { Randomised controlled trials. } \\
\text { If the number of RCTs meeting inclusion } \\
\text { criteria proves limited, we will broaden the } \\
\text { search strategy to include prospective and } \\
\text { retrospective cohort studies. }\end{array}$ & $\begin{array}{l}\text { Cross-sectional. } \\
\text { Case-control. } \\
\text { Case series with }<10 \text { patients. } \\
\text { Case reports. }\end{array}$ \\
\hline $\begin{array}{l}\text { Intervention } \\
\text { and } \\
\text { comparatort }\end{array}$ & $\begin{array}{l}\text { Full dose oral anticoagulation ( } \pm \text { antiplatelet } \\
\text { therapy) versus MAPT. } \\
\text { DPI (very low dose oral } \\
\text { anticoagulation+antiplatelet therapy) versus } \\
\text { MAPT. } \\
\text { DAPT versus MAPT. }\end{array}$ & $\begin{array}{l}\text { MAPT versus MAPT. } \\
\text { DAPT versus DAPT. } \\
\text { Anticoagulant versus anticoagulant. } \\
\text { MAPT versus placebo. } \\
\text { DAPT versus placebo. } \\
\text { Very low dose anticoagulation alone versus MAPT. }\end{array}$ \\
\hline Other & $\begin{array}{l}\text { There will be no limitation with respect to } \\
\text { language/date of publication. }\end{array}$ & $\begin{array}{l}\text { Trials focusing on intraoperative/intraprocedural } \\
\text { administration of anticoagulant therapy (eg, heparin } \\
\text { administered only during surgery and not extending into } \\
\text { the postoperative period), or on the acute management of } \\
\text { acute limb ischaemia (eg, thrombolytic therapy or therapies } \\
\text { lasting }<30 \text { days), will be excluded. }\end{array}$ \\
\hline
\end{tabular}

${ }^{*}$ In the event that a trial includes participants with PAD as a subgroup of the main trial, and sex-stratified outcomes are available for the overall trial but not within the PAD subgroup, then the sex-stratified outcomes from the main trial will be discussed in the narrative and quantitative synthesis (where applicable) and the population analysed will be clearly stated.

†There will be no limitation with respect to class/dosing of antithrombotic agents.

DAPT, dual antiplatelet therapy; DPI, dual pathway inhibition; MAPT, monoantiplatelet therapy; PAD, peripheral arterial disease; RCTs, randomised controlled trials. 


\section{Search strategy}

The search strategy will be designed with collaborative input from a librarian experienced in systematic review searches. A combination of Medical Subject Headings and keyword terms related to the population and interventions of interest will be used. The search strategy will begin with MEDLINE (see online supplemental appendix B) and will be translated according to the requirements of each electronic database. A search filter for RCTs will be applied.

\section{Study records}

Data management

Results from the literature search will be uploaded to Covidence online software. ${ }^{47}$ Duplicate articles will be identified and removed by the Covidence screen for duplicates and by the review authors. We will extract data from the final studies included in the narrative summary and quantitative synthesis-and perform statistical analysis-using RevMan 5.

\section{Selection process}

All titles, abstracts and full-text screening will be performed in duplicate by three independent reviewers. Studies that are disputed during the abstract review phase will be brought to full-text review. Disputes about full-text articles will be resolved through discussion between the reviewers and input from the study supervisors (PJ, DK and $\mathrm{MC}$ ) where required.

\section{Data collection process}

Data will be extracted from the studies by a team of independent reviewers and stored in a shared Excel document. Reviewers will undertake data collection in duplicate for the first few studies to ensure uniform data collection. Following this, data from each study will be collected independently and confirmed by a second reviewer. Any disputes will be resolved through discussion or input from the study supervisors.

\section{Data items}

The following data items will be extracted:

1. General study data: title, authors, year/journal/country/language of publication, sample size, recruitment period, study design, timing of randomisation to treatment, consideration for the potential impact of sex on the trial (ie, was the impact of sex analysed and how).

2. Population: baseline demographics (such as age, relevant comorbidities and medications), total number and proportion of females, total number and proportion of patients with symptomatic PAD (as well subcategories for severity of ischaemia or type of revascularisation where applicable), compliance with assigned treatment (and crossover rate in each arm).

3. Intervention: type, dosing, and frequency of antithrombotic, duration of therapy.

4. Comparator: type, dosing and frequency of antithrombotic, duration of therapy.
5. Outcomes: primary, secondary and safety outcomes, length of follow-up.

6. Outcome measures: reported effect measures for all outcomes, including $\mathrm{p}$ values, SD and CIs, time-toevent where applicable, analysis type (ie, intention to treat vs per protocol).

7. Funding: type and source where applicable.

\section{Outcomes and prioritisation}

Primary efficacy outcome

There will be two coprimary efficacy outcomes: a primary limb and a primary CV outcome. The primary limb outcome will be major (ie, above ankle) amputation. The primary CV outcome will be a composite of CV death, MI and stroke.

\section{Secondary efficacy outcomes}

Secondary outcomes will include the individual components of the primary CV outcome, acute limb ischaemia, surgical or endovascular intervention not planned at the time of randomisation and all-cause mortality, as well as primary, primary-assisted and secondary patency.

\section{Safety outcomes}

The primary safety outcome will be major bleeding as defined by each trial. The secondary safety outcome will be minor bleeding as defined by each trial. In trials that do not differentiate between major and minor bleeding, we will employ either the thrombolysis in myocardial infarction (TIMI) haemorrhage criteria or the International Society on Thrombosis and Hemostasis (ISTH) criteria (depending on the bleeding outcomes that were reported in the trial) to determine whether bleeding events should be classified as major or minor. TIMI classifies major bleeding as bleeding associated with any one of the following: 'is fatal, leads to hypotension requiring treatment with intravenous inotropic agents, requires surgical intervention for ongoing bleeding, necessitates the transfusion of four or more units of blood over a 48-hour period, or is a symptomatic intracranial hemorrhage'. ${ }^{48}$ ISTH defines major bleeding in non-surgical patients as 'fatal bleeding, and/or symptomatic bleeding in a critical area or organ....and/or bleeding causing a fall in hemoglobin level of $20 \mathrm{~g} / \mathrm{L}(1.24 \mathrm{mmol} / \mathrm{L})$ or more, or leading to transfusion of two or more units of whole blood or red cells'. ${ }^{49}$

\section{Risk of bias in individual studies}

Risk of bias will be assessed by one reviewer and verified by a second reviewer using the risk of bias tool (ROB 2) for randomised trials from the Cochrane Handbook for Systematic Reviews (p. 8) ${ }^{50}$ Using this tool, we will assess bias across several domains: randomisation, deviation from intended intervention, missing outcome data, outcome measurement and selective reporting. Results of the overall risk of bias assessment will be reported in a narrative summary and individual risk within each domain across all studies will be reported in a table. 
Sensitivity analysis will be performed excluding trials that demonstrate a low level of quality where applicable.

\section{Data synthesis}

We will assess patient population, study design, comparator arms and event rates for data synthesis. We will use $\mathrm{I}^{2}$ to assess for statistical heterogeneity between studies. A narrative synthesis will be reported instead of a metaanalysis if there is insufficient data to conduct a quantitative analysis. Pooled estimates will be calculated, and meta-analysis will be performed using RevMan V.5 software. Treatment effect will be measured using OR, risk difference or HR (depending on which measure is most commonly reported across included studies) to compare the effect of the antithrombotic intervention versus the MAPT control in females with symptomatic PAD. A random effects model will be employed to determine weights for meta-analysis.

The following preplanned subgroup analysis will be performed where data permits:

- Stratification by severity of symptoms: intermittent claudication versus critical limb ischaemia.

- Stratification by indication for therapy: PAD alone versus PAD plus coronary artery disease.

- Stratification by type of intervention: conservative versus open revascularisation (ie, bypass) versus endovascular revascularisation (ie, angioplasty and/or stenting) versus amputation.

We will conduct a sensitivity analysis excluding trials with high risk of bias or small number of participants. We will also conduct a sensitivity analysis excluding older trials if medical regimens beyond the assigned intervention and control arms vastly differ from more modern trials (eg, use of statin therapy). Meta-bias will be assessed by repeating the primary efficacy and safety outcomes using a fixed effects model and by creating a funnel plot if the number of included studies exceeds 10. If fewer than 10 studies are included in the final analysis, we will not evaluate for publication bias given its limited power in the setting of a small number of studies.

\section{Confidence in cumulative evidence}

The Grading of Recommendations Assessment, Development, and Evaluation framework will be employed to assess the quality of all reported outcomes. ${ }^{46}$

\section{Patient and public involvement}

Patients or the public were not involved in the design, or conduct, or reporting, or dissemination plans of our research.

\section{ETHICS AND DISSEMINATION}

Ethics approval is waived as the study does not involve primary data collection. This review will be submitted for publication in a peer-reviewed journal and for presentation at national and international scientific meetings.
Author affiliations

${ }^{1}$ Division of Vascular and Endovascular Surgery, Ottawa Civic Hospital, Ottawa, Ontario, Canada

${ }^{2}$ Department of Epidemiology, University of Ottawa, Ottawa, Ontario, Canada

${ }^{3}$ Department of Clinical Epidemiology, Ottawa Hospital Research Institute, Ottawa, Ontario, Canada

${ }^{4}$ Department of Internal Medicine, University of Ottawa Faculty of Medicine, Ottawa, Ontario, Canada

${ }^{5}$ Department of Medicine, The Ottawa Hospital, Ottawa, Ontario, Canada

Acknowledgements The authors would like to acknowledge Sarah Visintini, Berkman Library, University of Ottawa Heart Institute, for her assistance in designing our search strategy, and the PSI Foundation for their funding in support of medical trainee research.

Contributors All four authors substantially contributed to the design of this protocol. SAS drafted the protocol and is responsible for the design, execution and writeup of the study. PJ, DK and MC provided invaluable input in designing the study and will be overseeing the completion of this project. All four authors have provided approval of the version to be published and agree to be accountable for all aspects of the work, including its accuracy and integrity. SAS is the guarantor of the review.

Funding SAS was awarded the 2020 Physicians' Services Incorporated (PSI) Research Trainee Fellowship (Grant number RT4-2020) for a total of \$25 000 over 2 years to support clinically applicable research training opportunities.

Disclaimer The PSI foundation was not involved in developing any aspect of the protocol for this systematic review and meta-analysis.

Competing interests None declared.

Patient consent for publication Not required.

Provenance and peer review Not commissioned; externally peer reviewed.

Supplemental material This content has been supplied by the author(s). It has not been vetted by BMJ Publishing Group Limited (BMJ) and may not have been peer-reviewed. Any opinions or recommendations discussed are solely those of the author(s) and are not endorsed by BMJ. BMJ disclaims all liability and responsibility arising from any reliance placed on the content. Where the content includes any translated material, BMJ does not warrant the accuracy and reliability of the translations (including but not limited to local regulations, clinical guidelines, terminology, drug names and drug dosages), and is not responsible for any error and/or omissions arising from translation and adaptation or otherwise.

Open access This is an open access article distributed in accordance with the Creative Commons Attribution Non Commercial (CC BY-NC 4.0) license, which permits others to distribute, remix, adapt, build upon this work non-commercially, and license their derivative works on different terms, provided the original work is properly cited, appropriate credit is given, any changes made indicated, and the use is non-commercial. See: http://creativecommons.org/licenses/by-nc/4.0/.

ORCID iD

Shira A Strauss http://orcid.org/0000-0002-1614-1911

\section{REFERENCES}

1 Lovell M, Harris K, Forbes T, et al. Peripheral arterial disease: lack of awareness in Canada. Can J Cardiol 2009;25:39-45.

2 Criqui MH, Aboyans V, Criqui Michael H. Epidemiology of peripheral artery disease. Circ Res 2015;116:1509-26.

3 Criqui MH, Langer RD, Fronek A, et al. Mortality over a period of 10 years in patients with peripheral arterial disease. $N$ Engl J Med 1992;326:381-6.

4 Abu Dabrh AM, Steffen MW, Undavalli C, et al. The natural history of untreated severe or critical limb ischemia. $J$ Vasc Surg 2015;62:1642-51.

5 Meijer WT, Hoes AW, Rutgers D, et al. Peripheral arterial disease in the elderly. Arterioscler Thromb Vasc Biol 1998;18:185-92.

6 Stoffers HE, Rinkens PE, Kester AD, et al. The prevalence of asymptomatic and unrecognized peripheral arterial occlusive disease. Int J Epidemiol 1996;25:282-90.

7 Kannel WB, McGee DL. Update on some epidemiologic features of intermittent claudication: the Framingham study. J Am Geriatr Soc 1985;33:13-18. 
8 Fowkes FGR, Housley E, Cawood EHH, et al. Edinburgh artery study: prevalence of asymptomatic and symptomatic peripheral arterial disease in the general population. Int J Epidemiol 1991;20:384-92.

9 Murabito JM, Evans JC, Nieto K, et al. Prevalence and clinical correlates of peripheral arterial disease in the Framingham offspring study. Am Heart J 2002;143:961-5.

10 Hirsch AT, Allison MA, Gomes AS, et al. A call to action: women and peripheral artery disease. Circulation 2012;125:1449-72.

11 Jelani Q-ul-ain, Petrov M, Martinez SC, et al. Peripheral arterial disease in women: an overview of risk factor profile, clinical features, and outcomes. Curr Atheroscler Rep 2018;20.

12 Srivaratharajah K, Abramson BL. Women and peripheral arterial disease: a review of sex differences in epidemiology, clinical manifestations, and outcomes. Can J Cardiol 2018;34:356-61.

13 Collins TC, Suarez-Almazor M, Bush RL, et al. Gender and peripheral arterial disease. J Am Board Fam Med 2006;19:132-40.

14 McDermott MM, Greenland P, Liu K, et al. Sex differences in peripheral arterial disease: leg symptoms and physical functioning. J Am Geriatr Soc 2003;51:222-8.

15 Gardner AW. Sex differences in claudication pain in subjects with peripheral arterial disease. Med Sci Sports Exerc 2002;34:1695-8.

16 Egorova N, Vouyouka AG, Quin J, et al. Analysis of gender-related differences in lower extremity peripheral arterial disease. J Vasc Surg 2010;51:372-8.

17 Feinglass J, McDermott MM, Foroohar M, et al. Gender differences in interventional management of peripheral vascular disease: evidence from a blood flow laboratory population. Ann Vasc Surg 1994;8:343-9.

18 Rowe VL, Weaver FA, Lane JS, et al. Racial and ethnic differences in patterns of treatment for acute peripheral arterial disease in the United States, 1998-2006. J Vasc Surg 2010;51:S21-6.

19 Gutiérrez-Chico JL, Mehilli J. Gender differences in cardiovascular therapy: focus on antithrombotic therapy and percutaneous coronary intervention. Drugs 2013;73:1921-33.

20 Tangelder MJD, Algra A, Lawson JA, et al. Risk factors for occlusion of infrainguinal bypass grafts. Eur J Vasc Endovasc Surg 2000;20:118-24.

21 Gerhard-Herman MD, Gornik HL, Barrett C, et al. 2016 AHAV ACC guideline on the management of patients with lower extremity peripheral artery disease: Executive summary. Vasc Med 2017;22:NP1-43.

22 Aboyans V, Ricco J-B, Bartelink M-LEL, et al. Editor's choice - 2017 ESC guidelines on the diagnosis and treatment of peripheral arterial diseases, in collaboration with the European Society for Vascular Surgery (ESVS). Eur J Vasc Endovasc Surg 2018;55:305-68.

23 Mega JL, Simon T. Pharmacology of antithrombotic drugs: an assessment of oral antiplatelet and anticoagulant treatments. Lancet 2015;386:281-91.

24 Dabigatran - UpToDate. Available: https://www.uptodate.com/ contents/search?search $=$ dabigatran $\& \mathrm{sp}=0 \&$ searchType $=P L A I N$ TEXT\&source=USER_INPUT\&searchControl=TOP_PULLDOWN\& searchOffset $=1$ \&autoComplete $=$ true\&language $=\& \max =0$ \&index $=0$ 6\&autoCompleteTerm=dabig [Accessed 15 Dec 2020].

25 Rivaroxaban - UpToDate. Available: https://www.uptodate.com/ contents/search?search=rivaroxaban \&sp=0\&searchType $=$ PLAIN TEXT\&source=USER_INPUT\&searchControl=TOP_PULLDOWN\& searchOffset $=1$ \&autoComplete $=$ true\&language $=$ en $\&$ max $=10$ \&index $=$ 0 6\&autoCompleteTerm=rivaro [Accessed 15 Dec 2020].

26 Coppens M, Weitz JI, Eikelboom JWA. Synergy of dual pathway inhibition in chronic cardiovascular disease. Circ Res 2019:124:416-25.

27 Collaboration AT. Collaborative meta-analysis of randomised trials of antiplatelet therapy for prevention of death, myocardial infarction, and stroke in high risk patients. BMJ 2002;324:71-86.

28 Cacoub PP, Bhatt DL, Steg PG, et al. Patients with peripheral arterial disease in the charisma trial. Eur Heart J 2009;30:192-201.

29 Belch JJF, Dormandy J, et al, CASPAR Writing Committee. Results of the randomized, placebo-controlled clopidogrel and acetylsalicylic acid in bypass surgery for peripheral arterial disease (CASPAR) trial. J Vasc Surg 2010;52:825-33.
30 Bhatt DL, Flather MD, Hacke W, et al. Patients with prior myocardial infarction, stroke, or symptomatic peripheral arterial disease in the charisma trial. J Am Coll Cardiol 2007;49:1982-8.

31 Dörffler-Melly J, Koopman MMW, Prins MH. Antiplatelet and anticoagulant drugs for prevention of restenosis/reocclusion following peripheral endovascular treatment. Cochrane Database Syst Rev 2005:1:CD002071.

32 Strobl FF, Brechtel K, Schmehl J, et al. Twelve-month results of a randomized trial comparing mono with dual antiplatelet therapy in endovascularly treated patients with peripheral artery disease. $J$ Endovasc Ther 2013;20:699-706.

33 Tepe G, Bantleon R, Brechtel K, et al. Management of peripheral arterial interventions with mono or dual antiplatelet therapy-the MIRROR study: a randomised and double-blinded clinical trial. Eur Radiol 2012;22:1998-2006.

34 Warfarin Antiplatelet Vascular Evaluation Trial Investigators, Anand S, Yusuf S, et al. Oral anticoagulant and antiplatelet therapy and peripheral arterial disease. N Engl J Med 2007;357:217-27.

35 Efficacy of oral anticoagulants compared with aspirin after infrainguinal bypass surgery (the Dutch bypass oral anticoagulants or aspirin study): a randomised trial. Lancet 2000;355:346-51.

36 Sarac TP, Huber TS, Back MR, et al. Warfarin improves the outcome of infrainguinal vein bypass grafting at high risk for failure. $J$ Vasc Surg 1998;28:446-57.

37 Hussain MA, Al-Omran M, Creager MA, et al. Antithrombotic therapy for peripheral artery disease: recent advances. J Am Coll Cardiol 2018;71:2450-67.

38 Eikelboom JW, Connolly SJ, Bosch J, et al. Rivaroxaban with or without aspirin in stable cardiovascular disease. N Engl $\mathrm{J}$ Med 2017;377:1319-30.

39 xarelto-pm-en. Available: https://omr.bayer.ca/omr/online/xareltopm-en.pdf [Accessed 17 Mar 2021]

40 Bonaca MP, Bauersachs RM, Anand SS, et al. Rivaroxaban in peripheral artery disease after revascularization. N Engl J Med Overseas Ed 2020;382:1994-2004.

41 Conte MS, Bradbury AW, Kolh P, et al. Global vascular guidelines on the management of chronic limb-threatening ischemia. Eur $J$ Vasc Endovasc Surg 2019;58:S1-109.

42 Vyas MV, Mrkobrada M, Donner A, et al. Underrepresentation of peripheral artery disease in modern cardiovascular trials: systematic review and meta-analysis. Int J Cardiol 2013;168:4875-6.

43 Hoel AW, Kayssi A, Brahmanandam S, et al. Under-Representation of women and ethnic minorities in vascular surgery randomized controlled trials. J Vasc Surg 2009;50:349-54.

44 Rothwell PM, Goldstein LB. Carotid endarterectomy for asymptomatic carotid stenosis. Stroke 2004;35:2425-7.

45 Gerhard-Herman MD, Gornik HL, Barrett C, et al. 2016 AHA/ACC guideline on the management of patients with lower extremity peripheral artery disease: a report of the American College of Cardiology/American heart association Task force on clinical practice guidelines. J Am Coll Cardiol 2017;69:e71-126.

46 Moher D, Shamseer L, Clarke M, et al. Preferred reporting items for systematic review and meta-analysis protocols (PRISMA-P) 2015 statement. Syst Rev 2015;4:1.

47 Covidence - Better systematic review management. Available: https://www.covidence.org/home [Accessed 5 Jun 2020].

48 Wiviott SD, Antman EM, Gibson CM, et al. Evaluation of prasugrel compared with clopidogrel in patients with acute coronary syndromes: design and rationale for the trial to assess improvement in therapeutic outcomes by optimizing platelet inhibition with prasugrel thrombolysis in myocardial infarction 38 (TRITON-TIMI 38). Am Heart J 2006;152:627-35.

49 Schulman S, Kearon C, Subcommittee on Control of Anticoagulation of the Scientific and Standardization Committee of the International Society on Thrombosis and Haemostasis. Definition of major bleeding in clinical investigations of antihemostatic medicinal products in non-surgical patients. $J$ Thromb Haemost 2005;3:692-4.

50 Chapter 8: assessing risk of bias in a randomized trial. Available: / handbook/current/chapter-08 